http://jmscr.igmpublication.org/home/ ISSN (e)-2347-176x ISSN (p) 2455-0450 crossref DOI: https://dx.doi.org/10.18535/jmscr/v9i7.21

\title{
An observational study on use of Glasgow coma scale in neurology patients among staff nurses of the intensive care units and emergency departments in selected hospitals at Mangaluru
}

Journal Of Medical Science And Clinical Research

\author{
Authors \\ Mrs. Jonita Wilma Rodrigues ${ }^{1}$, Dr (Mrs) Larissa Martha Sams ${ }^{2}$ \\ ${ }^{1}$ MSc. Nursing, Medical Surgical Nursing, Laxmi Memorial College of Nursing, A.J Towers, Balmatta, \\ Mangalore, Karnataka, India \\ ${ }^{2}$ Professor and HOD, Department of Medical Surgical Nursing, Laxmi Memorial College of Nursing, \\ Mangalore, Karnataka, India
}

\begin{abstract}
Background: Glasgow coma scale (GCS) is a neurological scale which aims to give a reliable, objective way of recording the conscious state of person, for initial as well as subsequent assessment. The ultimate goal in the use of GCS is to identify those in need of acute intervention as early as possible and thus prevent secondary brain injuries. From a clinical and educational point of view, all health care professionals working in critical care setting should be competent to monitor and be equipped with clinical skills required that ensure high levels of patient safety and quality care.

Methodology: A descriptive survey approach was adopted to determine the use of Glasgow coma scale among the staff nurses of the intensive care units and emergency departments of selected hospitals at Mangalore. By purposive sampling, 60 samples were selected for the study. Data was collected from the sample by using Demographic and Clinical Proforma, Rating scale and Observational Checklist.

Results: There was a significant difference in the use of Glasgow coma scale among the nurses of Medical ICU, Neuro ICU and Emergency departments $(F=3.18, P, 0.05)$. Hence the null hypotheses $\left(H O_{1}\right)$ was rejected and research hypotheses was accepted. There was no significant association in use of GCS with the selected demographic variables of staff nurses. Hence the null hypotheses $\left(\mathrm{HO}_{2}\right)$ was accepted and research hypothesis was rejected..

Conclusion: From the findings of the study it can be concluded that majority of the nurses used the Glasgow coma scale appropriately.

Keywords: Observational Study, Glasgow coma scale, Neurology patients, Intensive care units, Emergency Departments.
\end{abstract}

\section{Introduction}

The level of consciousness is the sensitive and reliable indicator of a patient's neurological status. Assessment and documentation of the level of consciousness are considered as primary action for doctors and nurses who care for the patients with neurological or neurosurgical problems. ${ }^{1}$ The Glasgow Coma Scale (GCS) is the corner stone of the neurological assessment of patients used by both nursing and medical staff. ${ }^{2}$ The Glasgow
Coma Scale (GCS) was introduced in 1974 as a method for determining objectively the severity of brain dysfunction and coma six hours after the occurrence of head trauma ${ }^{3}$. The GCS is a key component of scoring systems, treatment protocols and general clinical decision making for critically ill patients. The GCS is a scaled assessment that measures the degree of consciousness under three distinct categories of neurological functioning, and each category is 
subdivided and given a score. It is an internationally recognized tool which assesses the conscious level of a patient with impaired consciousness. $^{4}$

The state of consciousness is characterized by the ability to get in contact with reality, to recognize objects that are part of it and interact with it. ${ }^{5}$. The cause of the alteration in consciousness is due to any traumatic brain injury. ${ }^{6}$ The altered conscious level can be further complicated by secondary insults to the central nervous system and this impaired conscious level may compound many other conditions ${ }^{4}$ Therefore when a patient is admitted in the critical care unit and emergency department of the hospitals it is very important to periodically check the neurological functioning of the nervous system.

The GCS is often incorporated to give continuous monitoring under standard conditions. ${ }^{3}$ This leads to a determination of a trend in the patient's condition, which can be more readily interpreted than by just using words and a description. So it is essential that the nurse should develop practice to conduct the neurological assessment with Glasgow Coma Scale competently and engage in the clinical reasoning for proper patient management.

\section{Materials and Method}

Descriptive obtrusive naturalistic observational research design is used in the study. Naturalistic observation design means covertly or overtly watching the subject's behaviour in their natural environment, without intervention. An investigator views the subjects as it takes place. The observation may be either direct, where the investigator is actually present during the task, or indirect, where the task is viewed by some other means such as through the use of video camera. Demographic and Clinical Proforma,Rating scale to assess the use of Glasgow Coma Scale among Intensive care units and Emergency department nurses in neurology patients, Observational Checklist for Validating Documentation of GCS.

\section{Results}

Table 1: Description of sample according to demographic variables $\mathrm{N}=60$

\begin{tabular}{|c|c|c|}
\hline $\begin{array}{l}\text { Sl. } \\
\text { No. }\end{array}$ & Demographic variables & $\mathbf{f}(\%)$ \\
\hline \multirow[t]{7}{*}{1.} & Age (in years) & \\
\hline & $21-25$ & $35(58.3)$ \\
\hline & $26-30$ & $18(30)$ \\
\hline & $31-35$ & $6(10)$ \\
\hline & $36-40$ & $1(1.7)$ \\
\hline & $41-45$ & - \\
\hline & $>45$ & - \\
\hline \multirow[t]{3}{*}{2.} & Gender & \\
\hline & a. Male & $7(11.7)$ \\
\hline & Female & $53(88.3)$ \\
\hline \multirow[t]{5}{*}{3.} & Qualification & \\
\hline & a. Diploma in Nursing & $14(23.3)$ \\
\hline & Basic Nursing & $37(61.7)$ \\
\hline & Post Basic nursing & $9(15)$ \\
\hline & M. Sc. Nursing & - \\
\hline \multirow[t]{3}{*}{4.} & Job status & \\
\hline & Junior staff nurse & $28(46.7)$ \\
\hline & Senior staff nurse & $32(53.3)$ \\
\hline \multirow[t]{4}{*}{5.} & Years of Nursing Experience & \\
\hline & a. $\quad 1-5$ & $51(85)$ \\
\hline & $6-10$ & $9(15)$ \\
\hline & $>10$ & \\
\hline \multirow[t]{3}{*}{6.} & Have you used GCS previously & \\
\hline & a. Yes & $60(100)$ \\
\hline & No & - \\
\hline
\end{tabular}


Table 2: Percentage Distribution of subjects according to the use of Glasgow coma scale by using rating scale

\begin{tabular}{|c|c|c|c|c|}
\hline Items & & $\begin{array}{l}\text { Always } \\
\text { f \% }\end{array}$ & $\begin{array}{l}\text { Sometimes } \\
\text { f \% }\end{array}$ & $\begin{array}{l}\text { Never } \\
\text { f \% }\end{array}$ \\
\hline 1. & $\begin{array}{l}\text { Intensive care unit/emergency department nurses uses Glasgow } \\
\text { Coma Scale to assess the level of consciousness of his/her patient }\end{array}$ & 60100 & - & - \\
\hline 2. & $\begin{array}{l}\text { Checks all the } 3 \text { components of the Glasgow Coma Scale during } \\
\text { every assessment }\end{array}$ & 60100 & - & - \\
\hline \multirow[t]{5}{*}{ A. } & Eye opening & & & \\
\hline & $\begin{array}{l}\text { - Goes near the patient and observes if patient opens the } \\
\text { eyes }\end{array}$ & 60100 & - & - \\
\hline & $\begin{array}{l}\text { - Calls and commands the patient and observes if the } \\
\text { patient opens the eyes }\end{array}$ & 60100 & - & - \\
\hline & $\begin{array}{l}\text { - Applies pressure on limbs and observes if patient } \\
\text { responds to pain }\end{array}$ & 2745.0 & 3355.0 & - \\
\hline & $\begin{array}{l}\text { - Applies supraorbital pressure and observes if patient } \\
\text { responds }\end{array}$ & 5185.0 & 915.0 & - \\
\hline \multirow[t]{7}{*}{ B. } & Motor responses & & & \\
\hline & $\begin{array}{l}\text { - Gives patient simple commands and observes if patient } \\
\text { responds }\end{array}$ & 60100 & - & - \\
\hline & $\begin{array}{l}\text { Gives painful stimuli and observes if patient uses arm } \\
\text { and attempts to remove pressure }\end{array}$ & 5388.3 & 711.7 & - \\
\hline & Observes if there is flexion of arm in pain & 5286.7 & 813.3 & - \\
\hline & Observes if there is flexion of arm in response to pain & 5388.3 & 711.7 & - \\
\hline & $\begin{array}{l}\text { Observes if patients shoulders are adducted and } \\
\text { shoulder is internally rotated and forearm is pronated }\end{array}$ & 4778.3 & 1321.7 & - \\
\hline & Observes if the limbs remains flaccid & 1016.7 & 2948.3 & 2135.0 \\
\hline \multirow[t]{6}{*}{ C. } & Verbal response & & & \\
\hline & $\begin{array}{l}\text { - Talks to the patient, and observes if the patient } \\
\text { converses and is oriented }\end{array}$ & 4270 & 1830 & - \\
\hline & $\begin{array}{l}\text { Observes if the patient converses and is confused and } \\
\text { disoriented }\end{array}$ & 5795.0 & 35.0 & - \\
\hline & $\begin{array}{l}\text { Observes if the patient uses intelligible but no sustained } \\
\text { sentences }\end{array}$ & 4473.3 & 1626.7 & - \\
\hline & $\begin{array}{l}\text { Observes if the patient moans and groans and there is no } \\
\text { speech }\end{array}$ & 5896.7 & 23.3 & - \\
\hline & Observes if there is no verbalization & 5998.3 & 11.7 & - \\
\hline 3. & Reassessment of the Glasgow coma as per protocol & 5998.3 & 11.7 & \\
\hline 4. & Glasgow Coma Scale documentation is done as per protocol & 60100 & - & - \\
\hline 5. & Glasgow coma documentation sites maintained & 60100 & - & - \\
\hline 6. & Glasgow Coma Scale is communicated verbally to the manager & 46.7 & 5693.3 & - \\
\hline 7. & Proficient in using Glasgow Coma Scale in all neurological cases & 5286.7 & 813.3 & - \\
\hline 8. & Takes more time to assess the Glasgow Coma Scale & . & 1118.3 & 4981.7 \\
\hline
\end{tabular}

Table 3: Percentage distribution of Documentation of Glasgow Coma Scale (GCS) using observational checklist

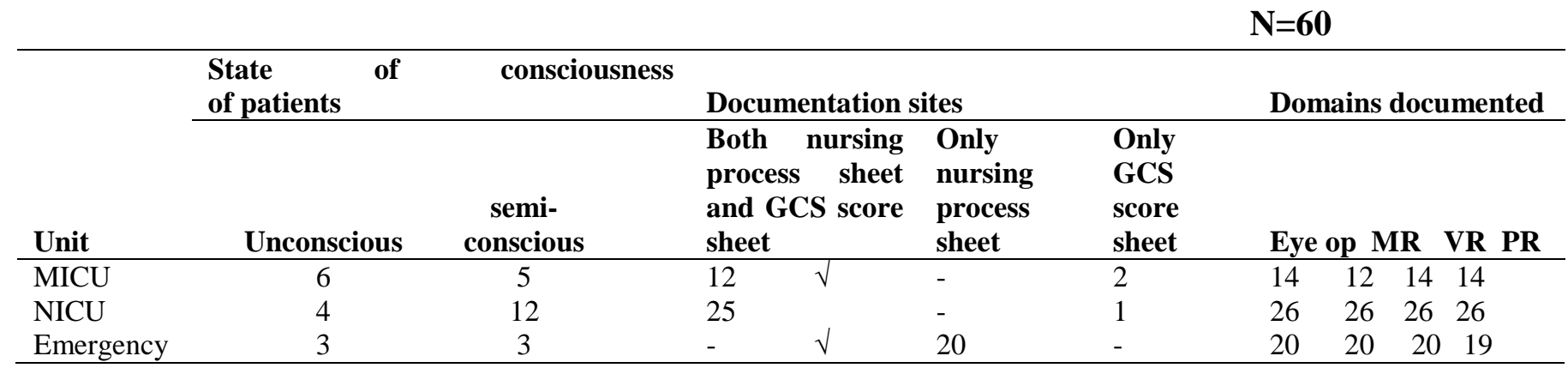

$\mathrm{MR}=$ motor response, $\mathrm{VR}=$ verbal response, $\mathrm{PR}=$ pupillary reaction. 
Table 4: Difference in the use of Glasgow Coma Scale among the nurses Of MICU, Neuro ICU and emergency departments (rating scale)

\begin{tabular}{lccccc} 
& & & & N=60 \\
\hline Between Groups & Sum of squares & df & Mean square & F & p value \\
Within Groups & 18.065 & 2 & 9.033 & 5.315 & $0.008^{* *}$ \\
\hline Total & 96.868 & 57 & 1.699 & & \\
\hline $\mathrm{F}_{(2,57)}=3.18, \mathrm{p}<0.05, * *=$ Highly significant & & & & &
\end{tabular}

Table 5: Association in use of GCS with the selected demographic and clinical variables of staff nurses

\begin{tabular}{lcc} 
& & $\mathbf{N}=60$ \\
\hline Demographic factors & $\chi^{\mathbf{2}}$ value & p value \\
\hline Age & 1.099 & 0.777 \\
Gender & 0.615 & 0.433 \\
Qualification & 0.879 & 0.644 \\
Job status & 0.950 & 0.330 \\
Experience & 0.431 & 0.511 \\
Use of GCS & 0.882 & 0.830 \\
\hline$\chi^{2}{ }_{1}=3.84, \chi^{2}=5.99, \chi^{2}{ }_{4}=9.48, \chi^{2}{ }_{6}=12.59 ; \mathrm{p}<0.05$ &
\end{tabular}

\section{Discussion}

\section{Description of Demographic Characteristics of Sample}

In the present study, majority of sample $(58.3 \%)$ were in the age group of 21-25 years and females (88.3\%). Most of samples (61.7\%) had completed B. Sc. Nursing. Most of the subjects (53.3\%) were senior staff nurses. A study conducted to explore nurses knowledge in using the Glasgow Coma Scale in an Acute care hospital, Singapore showed that most of subjects (54\%) were senior staff nurses. $^{14}$

Highest percentage of subjects $(85.0 \%)$ were having 1-5 years of nursing experience in the intensive care unit and emergency department and all subjects were using the Glasgow Coma Scale previously for neuro assessment. A study on effectiveness of self instructional module on knowledge and skills regarding use of Glasgow Coma Scale in neurological assessment of patients among nurses working in Critical care units of KLE Hospital, Belgaum which showed that majority of subjects $(80 \%)$ had $0-2$ year experience in the critical care unit ${ }^{48}$. A study conducted on assessment of nurse's knowledge about Glasgow Coma Scale at a university hospital, USA also showed majority of nurses $(89.8 \%)$ had used the Glasgow Coma Scale previously in the assessment of neurology patients. ${ }^{13}$

\section{Description of use of Glasgow Coma Scale in intensive care units and emergency department nurses using rating scale}

The present study showed that all the subjects always use the Glasgow Coma Scale (GCS) in the intensive care unit and emergency department and checked all the 3 components during every assessment. In eye opening, all the subjects always went near the patient and observed if patient opens the eyes and given commands to the patient and observed if the patient opened the eyes. In the motor response, all the subjects always gave patient simple commands and observed if patient responds. Most of the samples (35\%) never checked if the patients limbs are flaccid. In the verbal response. Majority of the sample (95\%) observed if the patient converses and is confused and (96.7\%) always observed if there is no verbalization of any type.

Majority of sample (98.3\%) always did reassessment of the Glasgow Coma Scale as per protocol. All the subjects always did Glasgow Coma Scale documentation as per the protocol and maintained the Glasgow Coma Scale documentation sites .Highest percentage of subjects $(86.7 \%)$ were proficient in using Glasgow Coma Scale in all neurological cases. Majority of sample $\quad(93.3 \%)$ sometimes communicated 
Glasgow Coma Scale verbally to the manager. Most of the subjects (81.7\%) never took more time to assess the Glasgow Coma Scale.

In a study to evaluate the accuracy of Glasgow com scale Knowledge and Performance among Vietnamese nurses, showed that majority of subjects $(64 \%)$ had correct performance on eye opening. Highest percentage $(75.5 \%)$ of the subjects had correct performance on verbal response. Majority of the subjects $(78.7 \%)$ had correct performance on motor response.

\section{Validation of use of Glasgow Coma Scale in Intensive care units and emergency department nurses using observational checklist}

In the present study, on observation, there were 6 unconscious patients and 5 semiconscious patients in Medical ICU, 4 unconscious and 12 semiconscious patients in Neuro ICU,3 Unconscious and 3 semiconscious patients in emergency department. On observing the documentation sites, In Medical ICU, out of 14 Patient case files maintained by the subjects and observed by investigator, 12 case files were having documentation of GCS in both Nursing process sheet and GCS score sheet and 2 case files were having only GCS score sheet whereas in Neuro ICU, out of 26 case files maintained by the subjects and observed by the investigator, 25 case files were having documentation of GCS scores in both Nursing process sheet and GCS score sheet and 1 case file was having only GCS score sheet. In emergency department, out of 20 case files maintained by subject, GCS score was maintained only in the nursing process sheet. In the domains documented, all the ICU and emergency department case files had domains recorded except for pupillary reaction in 1-2 files.

A similar study was done on nurse's knowledge of Glasgow Coma Scale in neurological assessment of patients in a selected tertiary hospital, Nigeria where a similar observational checklist was maintained which showed that there were 6 unconscious patients during observation and there were 6 files with GCS data in the Neuro ward.
Documentation was done in nursing process sheet in Neuro ICU .There was no documentation of pupillary reaction to light in the case files. There were 3 unconscious patients in Emergency department. Documentation was done using nursing process sheet and handover note book. No record was found for pupillary reaction to light in the emergency unit in all the 3 case files. In the ICU, there were 5 unconscious patients. Documentation was done using GCS Score sheet .In the domains, Eye opening, verbal response, motor response was maintained whereas the pupillary reaction to light domain not maintained. ${ }^{12}$

Difference in the use of Glasgow Coma Scale among the nurses Of MICU, Neuro ICU and emergency departments (rating scale)

There was significant difference in the use of Glasgow Coma Scale among the nurses Of MICU, Neuro ICU and emergency departments was found significant (F value, $\left.\mathrm{F}_{(2,57)}=3.18, \mathrm{p}<0.05\right)$. Thus the null hypotheses was rejected and research hypotheses was accepted.

A study was done on nurses knowledge of Glasgow Coma Scale in neurological assessment of patients in selected tertiary hospital, Nigeria showed that there was significant differences in all items related to nurses' usage of the GCS between the various wards/units. $(\mathrm{p}=0.008) .{ }^{12}$

\section{Association of the use of GCS with the selected demographic and clinical variables of staff nurses}

The findings of the present study showed there was no significant association in use of GCS with the selected demographic variables of staff nurses. These findings were supported by study on nurses knowledge of Glasgow Coma Scale in neurological assessment of patients in selected tertiary hospital, Nigeria showed that there was no significant association between the nurses gender, age, level of experience and knowledge on usage of GCS. ${ }^{12}$ 


\section{Conclusion}

There was a significant difference in the utilization of Glasgow Coma scale among the nurses of the intensive care units and emergency departments. There was no significant association in use of GCS with the selected demographic and clinical variables of staff nurses.

\section{Limitations}

1. The study was confined to a small sample selected by purposive sampling technique which restricts the generalization.

2. The study was limited to staff nurses working in only critical care units and emergency departments at selected hospitals in Mangaluru.

\section{Recommendations}

Based on the study findings, the following recommendations are stated:

- A similar study can be replicated with a large sample in order to generalize the findings.

- $\quad$ Comparative studies can be conducted in different settings.

- The research tool can be updated and can be used as evaluation criteria for the nursing working skills in the critical care units and emergency department.

\section{Acknowledgement}

The author would like to express heartfelt thanks with deep sense of gratitude and respect to guide Dr. Mrs. Larissa Martha Sams, Principal and HOD, Department of Medical and Surgical Nursing, Laxmi memorial college of nursing,

\section{Conflict of Interest: Nil}

Source of Funding: Nil

Ethical Clearance: The ethical clearance for the present study was obtained from A.J Ethics Committee, A.J Hospital and Research Centre, Mangaluru.

\section{Bibliography}

1. Lewis $\mathrm{S}$, Heitkemper $\mathrm{M}$, Dirksen $\mathrm{S}$. Medical surgical nursing: assessment and management of clinical problems. $7^{\text {th }}$ ed. 2007; 1442.

2. Dowsen S. Brain and nervous system. 2010. Available from: URL: http://kidshealth.org/parent /body_ basics/. nervous system.html.

3. Edwards S. Using the Glasgow Coma Scale: analysis and limitations. British Journal of Nursing 2001;10(2):92-101.

4. Palmer R, Knight J. Assessment of altered conscious level in clinical practice. British Journal of Nursing 2006;15(22):1255-9.

5. Bordini AL, Luiz TF, Fernandes $M$. Historical Review and Neuropsychiatry 2010;68(6):930-7.

6. Traumatic brain injury information. National Institute of Neurological Disorders and Stroke. Available from: URL:http://www.ninds.nih.gov/disorders/t bi.htm

7. Black JM, Hawks JH. Medical surgical nursing : clinical management for positive

8. Weller FB. Neurological evaluation techniques. Nurse's Dictionary.

9. McNarry AF, Goldhill DR. Simple bedside assessment of level of consciousness: comparison of two simple assessment scales with the Glasgow Coma Scale. 2004 Jan;59(1):34-7.

10. Matis G, Birbilis. The Glasgow Coma scale - a brief review, 2008. Available from:

URL:

http//www.mendley.com/research/Glasgo w Coma Scale

11. Heim C, Schoetker P, Spahn DR. Glasgow Coma Scale in traumatic brain injury 2004 Dec;53(12):1245-55.

12. Ogunfowokan AA, Olaogun AA, Okorodudu TU. Evaluation of clinical nurses' use of Glasgow Coma Scale in selected Teaching Hospital in Osun State 
Nigeria. West African Journal of Nursing 2010;21(2):87-92.

13. Glasgow Coma Scale. Wikipedia. Available from: URL: http://en.wikipedia.org/wiki/Glasgowcoma

14. Hickey JV. The clinical practice of neurological and neurosurgical nursing. $4^{\text {th }}$ ed. Philadelphia: J. B. Lippincott Company; 1997. p. 133-61.

15. Ihsan M, Sok YL, Moon FC. A study to explore nurses knowledge in using the Glasgow Coma Scale in an acute care hospital. Journal of Neuroscience Nursing; 45(5):272-80. 